\title{
Multi-pulse Orbits and Homoclinic Trees in a Non-autonomous Resonant Hamiltonian System
}

\author{
Sha Zhou ${ }^{1,2}$, Wei Zhang ${ }^{1.2}$ and Tian-jun $\mathrm{Yu}^{1.2}$ \\ ${ }^{1}$ Beijing Key Laboratory of Nonlinear Vibrations and Strength of Mechanical Structures, 100124,China \\ ${ }^{2}$ Beijing University of Technology, College of Mechanical Engineering, 100124, China
}

\begin{abstract}
In this study, we develop the energy-phase method to deal with the high-dimensional non-autonomous nonlinear dynamical systems. Our generalized energy-phase method applies to integrable, two-degree-of freedom non-autonomous resonant Hamiltonian systems. As an example, we investigate the multi-pulse orbits and homoclinic trees for a parametrically excited, simply supported rectangular thin plate of two-mode approximation. In both the Hamiltonian and dissipative case we find homoclinic trees, which describe the repeated bifurcations of multi-pulse solutions, and we present visualizations of these complicated structures.
\end{abstract}

\section{Introduction}

The energy-phase method, which was first presented by Haller and Wiggins [1-10], was used to reveal families of multi-pulse solutions and predict the parameter region for chaotic motion may occur.

Although the energy-phase method has been applied widely to engineering problems, it was used to solve autonomous perturbed Hamiltonian systems. It is worth to mention that the energy-phase method has never used in non-autonomous nonlinear dynamical systems. In this paper, we develop the energy-phase method to deal with non-autonomous nonlinear dynamical systems for the first time.

This paper develops the energy-phase method to deal with the high-dimensional non-autonomous nonlinear dynamical systems. In section 2 , we formulate the problem and describe the geometrical structure of the phase space of the unperturbed systems, and we study the dynamics in the perturbed system, and derive the explicit formulas of the nth order energy difference function for the non-autonomous systems. In section 3 , we apply our developed methods to a specific example: a two-mode truncation of parametrically excited, simply supported rectangular thin plate. And we visualize the homoclinic tree and show by explicit calculations how it breaks up under the effect of dissipation. We make a conclusion in section 4 .

\section{Generalized of the energy-phase method}

Let us consider a two-degree-of -freedom Hamiltonian systemgiven by

$$
\begin{gathered}
\dot{x}=J D_{x} H_{0}(x, I)+\varepsilon J D_{x} H_{1}(x, I, \gamma, \omega t ; \varepsilon)+\varepsilon g^{x}(x, I, \gamma, \phi ; \varepsilon) \\
\dot{I}=-\varepsilon D_{\gamma} H_{1}(x, I, \gamma, \omega t ; \varepsilon)+\varepsilon g^{I}(x, I, \gamma, \phi ; \varepsilon) \\
\dot{\gamma}=D_{I} H_{0}(x, I)+\varepsilon D_{I} H_{1}(x, I, \gamma, \omega t ; \varepsilon)+\varepsilon g^{\gamma}(x, I, \gamma, \phi ; \varepsilon) \\
\dot{\phi}=\omega \\
0 \leq \varepsilon<1,(x, I, \gamma, \phi) \in \mathbf{R}^{2} \times \mathbf{U} \times \mathbf{S}^{1} \times \mathbf{S}^{1}
\end{gathered}
$$

where $\mathbf{U} \subset \mathbf{R}^{+}$is an open set, functions $H_{0}$ and $H_{1}$ are $\mathrm{C}^{r+1}(r \geq 2)$ functions, and functions $H_{1}$ and $g=\left(g^{x}, g^{I}, g^{\gamma}\right)$ are periodic in $t$ with the period $T=2 \pi / \omega$, and

$$
J=\left(\begin{array}{cc}
0 & 1 \\
-1 & 0
\end{array}\right) .
$$

Note that when $\varepsilon=0$ system (1) is an integrable Hamiltonian system on which we make two structural as sumptions:

(H1) There exist $I_{1}, I_{2} \in \mathbf{U}, I_{1}<I_{2}$ such that for any $I \in\left[I_{1}, I_{2}\right]$,

(1) has a hyperbolic fixed point $\bar{x}_{0}(I)$ and a homoclinic trajectory $x^{h}(t, I)$, which connects $\bar{x}_{0}(I)$ to itself.

(H2) (Resonance) There exists $I_{r} \in\left(I_{1}, I_{2}\right)$ such that

$$
\begin{gathered}
D_{I} H_{0}\left(\bar{x}_{0}\left(I_{r}\right), I_{r}\right)=0 ; \\
\left.D_{I}^{2}\left[H_{0}\left(\bar{x}_{0}(I), I\right)\right]\right|_{I=I_{r}} \neq 0 .
\end{gathered}
$$

Defining a cross-section in full five-dimensional phase space of equation (2), we have 


$$
\Sigma^{\phi_{0}}=\left\{(x, I, \gamma, \phi) \mid \phi=\phi_{0}\right\}
$$

Figure 1 gives the geometrical explanation of the crosssection $\Sigma^{\phi_{0}}$.

Let $q(t)=(x(t), I(t), \gamma(t)), \quad(q(t), \phi(t))$ and $\left(q_{\varepsilon}(t), \phi(t)\right)$ be trajectories of the unperturbed and perturbed vector fields, respectively.

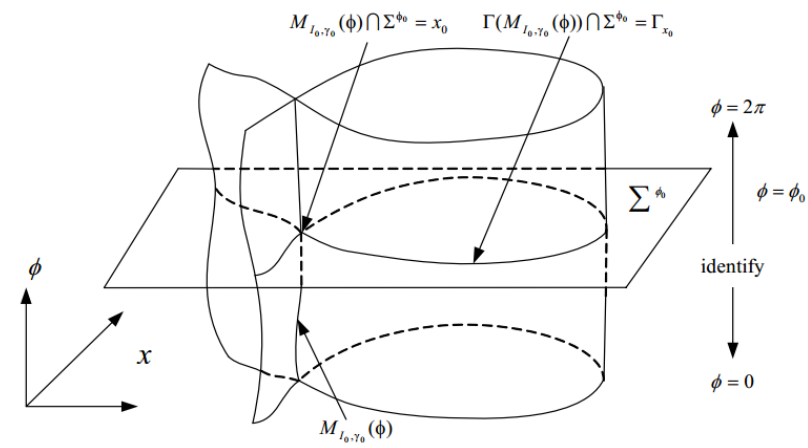

Figure 1 Geometrical structure of the cross-section $\Sigma^{\phi_{0}}$

Let $\left(q_{\varepsilon}^{s}(0), \phi_{0}\right)=s_{1},\left(q_{\varepsilon}^{u}(0), \phi_{0}\right)=p_{1}$. If $p_{1}$ and $s_{1}$ coincide, there exists single-pulse homoclinic orbits

And $p_{1}$ and $s_{1}$ coincide if

$$
\frac{1}{\varepsilon}\left[H\left(s_{1}, \varepsilon\right)-H\left(p_{1}, \varepsilon\right)\right]=0
$$

And the energy difference function can be written as

$$
\varepsilon \Delta^{1} H\left(\phi_{0}\right)=H\left(s_{1}, \varepsilon\right)-H\left(p_{1}, \varepsilon\right)
$$

Therefore

$$
\begin{gathered}
\Delta^{1} H\left(t, \phi_{0}\right)=H_{D}\left(h, \gamma+\Delta \gamma, \phi_{0}\right)-H_{D}\left(h, \gamma, \phi_{0}\right)-\int_{-\infty}^{\infty} \omega D_{\phi} H_{D}(h, \gamma, \phi) d s \\
-\left.\int_{-\infty}^{\infty}\left\langle D H_{0}(x, I), g(x, I, \gamma, \phi)\right\rangle\right|_{x^{1}(t)} d t
\end{gathered}
$$

It is known that $g(x, I, \gamma, \phi)$ is bounded for all $\tau$ and $D H_{0}(x, I)$ goes to zero exponentially fast as $\tau \rightarrow \pm \infty$ since $x^{1}(t)$ approaches a hyperbolic fixed point. Therefore, the improper integral $\left.\quad \int_{-\infty}^{\infty}\left\langle D H_{0}(x, I), g(x, I, \gamma, \phi)\right\rangle\right|_{x^{1}(t)} d t$ converges absolutely. And $\int_{-\infty}^{\infty} \omega D_{\phi} H(h, \gamma, \phi) d t$ converges at best conditionally. The improper integral makes sense if we approach the limits $\pm \infty$ along the sequences of times $\left\{\tau_{j}^{s}\right\},\left\{\tau_{j}^{u}\right\}, j=1,2, \cdots$.

Hence, for $\varepsilon$ small enough, if

$$
\Delta^{1} H\left(\gamma, \phi_{0}\right)=0, D \Delta^{1} H\left(\gamma, \phi_{0}\right) \neq 0
$$

there exist 1-pulse homoclinic orbits.

To formulate the above algorithm concisely, we introduce the energy difference function

$$
\begin{gathered}
\Delta^{N} H\left(\phi_{0}\right)=H_{D}\left(h, \gamma+N \Delta \gamma, \phi_{0}\right)-\sum_{j=0}^{N-1} \int_{-\infty}^{\infty} \omega D_{\phi} H_{D}(h, \gamma+j \Delta \gamma, \phi) d s \\
-H_{D}\left(h, \gamma, \phi_{0}\right)-\left.\sum_{i=1}^{N} \int_{-\infty}^{\infty}\left\langle D H_{0}(x, I), g(x, I, \gamma, \phi)\right\rangle\right|_{x^{i}(t)} d t
\end{gathered}
$$

If equation (6) has transverse zero, then there exist $N$ pulse homoclinic orbit.

\section{Equations of motion of the buckled thin plate}

We consider the simply supported at the four-edge rectangular thin plate whose edge lengths are $a$ and $b$ and thickness is $h$. The thin plate is subjected to its plane excitations. We establish a Cartesian coordinate system shown in Figure 2 and the coordinate $O x y$ is located at the middle surface of the thin plate. It is assumed that $u$, $v$ and $w$ represent the displacement of a point in the middle plane of the thin plate in the $x, y$ and $z$ directions, respectively. The in -plate excitation of the thin plate may be expressed in the form $p=p_{0}-p_{1} \cos \Omega t$.

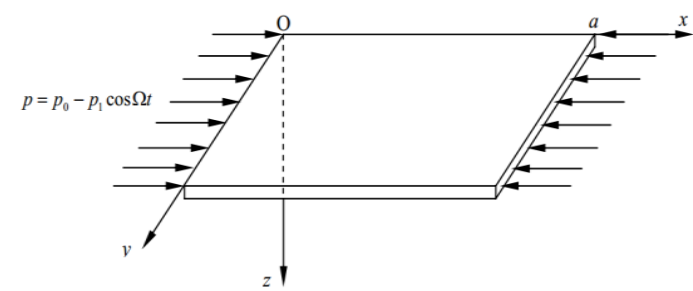

Figure 2 The model of rectangular thin plate and the coordinate system

According to ref [11], the dimensionless equations of motion for the thin plate under parametric excitation are obtained as follows

$$
\begin{aligned}
& \ddot{x}_{1}+\mu \dot{x}_{1}-g_{1} x_{1}+2 x_{1} f_{1} \cos \Omega t+\alpha_{1} x_{1}^{3}+\alpha_{2} x_{1} x_{2}^{2}=0 \\
& \ddot{x}_{2}+\mu \dot{x}_{2}-g_{2} x_{2}+2 x_{2} f_{2} \cos \Omega t+\beta_{1} x_{2}^{3}+\beta_{2} x_{2} x_{1}^{2}=0
\end{aligned}
$$

Using the normal form theory and the canonial transformation, equation (1) can be transformed to

$$
\begin{gathered}
\dot{y}_{1}=\frac{\partial H_{0}}{\partial y_{2}}+\varepsilon \frac{\partial H_{1}}{\partial y_{2}}+\varepsilon g^{y_{1}}=y_{2} \\
\dot{y}_{2}=-\frac{\partial H_{0}}{\partial y_{1}}-\varepsilon \frac{\partial H_{1}}{\partial y_{1}}+\varepsilon g^{y_{2}} \\
=g_{2} y_{1}-\varepsilon \mu y_{2}-\beta y_{1}^{3}-\beta_{2} y_{1} I-2 \varepsilon y_{1} f_{2} \cos \phi \\
\dot{I}=\frac{\partial H_{0}}{\partial \gamma}+\varepsilon \frac{\partial H_{1}}{\partial \gamma}+\varepsilon g^{I} \\
=-\varepsilon \mu I-2 \varepsilon f I \sin 2 \gamma \cos \phi \\
\dot{\gamma}=-\frac{\partial H_{0}}{\partial I}-\varepsilon \frac{\partial H_{1}}{\partial I}+\varepsilon g^{\gamma} \\
=-b-\varepsilon f(\cos 2 \gamma+1) \cos \phi-\frac{3}{4} \alpha I-\frac{1}{2} \alpha_{2} y_{1}^{2}
\end{gathered}
$$




$$
\dot{\phi}=\Omega
$$

where

$$
\begin{aligned}
& H_{0}\left(y_{1}, y_{2}, I\right)=\frac{y_{2}^{2}}{2}-\frac{g_{2}}{2} y_{1}^{2}+\frac{\beta}{4} y_{1}^{4}+\frac{\beta_{2}}{2} y_{1}^{2} I+b I+\frac{3}{8} \alpha I^{2} \\
& H_{1}\left(y_{1}, y_{2}, I, \gamma, \phi\right)=y_{1}^{2} f_{2} \cos \phi+f I \cos 2 \gamma \cos \phi+f I \cos \phi
\end{aligned}
$$

and $g^{y_{1}}, g^{y_{2}}, g^{I}$ and $g^{\gamma}$ are the perturbation terms induced by the dissipative effects

$$
g^{y_{1}}=0, g^{y_{2}}=-\mu y_{2}, g^{I}=-\mu I, g^{\gamma}=0 .
$$

\subsection{The unperturbed dynamics}

When $\varepsilon=0$, it is noted that system (8) is an uncoupled two-degree-of-freedom nonlinear system since $\dot{I}=0$. Consider the first two decoupled equations

$$
\begin{gathered}
\dot{y}_{1}=y_{2} \\
\dot{y}_{2}=g_{2} y_{1}-\beta y_{1}^{3}-\beta_{2} y_{1} I
\end{gathered}
$$

Since $\beta>0$, system (11) can exhib it the homoclinic bifurcation on the curve defined by $g_{2}=\beta_{2} I$. When $g_{2}-\beta_{2} I<0$, the trivial zero solution may bifurcate into three solutions through pitchfork bifurcation, which are given by $q_{0}=(0,0)$ and $q_{ \pm}(I)=(B, 0)$, respectively, where

$$
B= \pm \sqrt{\frac{g_{2}-\beta_{2} I}{\beta}}
$$

From the Jacobian matrix evaluated at the non-zero solutions, it is known that the singular point $q_{0}=(0,0)$ is the saddle point and the singular points $q_{ \pm}(I)=(B, 0)$ are center points.

Thus, for all $I \in\left[I_{1}, I_{2}\right]$, system (11) has one hyperbolic saddle point $q_{0}$ which is connected to a pair of homoclinic orbits $\lim _{T_{1} \rightarrow \pm \infty} y_{ \pm}^{h}\left(T_{1}, I\right)=q_{0}$. Letting $\eta=g_{2}-\beta_{2} I$, then the homoclinic orb its can be obtained as follows

$$
\begin{gathered}
y_{1}\left(T_{1}\right)= \pm \sqrt{\frac{\eta}{\beta}} \sec h\left(\sqrt{\eta} T_{1}\right) \\
y_{2}\left(T_{1}\right)=\mp \frac{\sqrt{2} \eta}{\sqrt{\beta}} \tanh \left(\sqrt{\eta} T_{1}\right) \sec h\left(\sqrt{\eta} T_{1}\right)
\end{gathered}
$$

Therefore, in the full five-dimensional phase space, the set defined by

$$
M=\left\{(y, I, \gamma, \phi) \mid y=q_{0}=(0,0), I \in\left[I_{1}, I_{2}\right], \gamma \in[0,2 \pi), \phi=\Omega t+\phi_{0}\right\}
$$

is a three dimensional invariant manifold.

Considering the unperturbed system of equation (8) restricted to $M$, we can calculate the resonant value

$$
I_{r}=\frac{-4 b}{3 \alpha}
$$

and the phase shift $\Delta \gamma$ of the oscillations is defined as

$$
\Delta \gamma=\gamma\left(+\infty, I_{r}\right)-\gamma\left(-\infty, I_{r}\right)=-\frac{2 \alpha_{2}}{\beta} \sqrt{\eta}
$$

\subsection{The perturbed dynamics}

It is noticed that the saddle point may persist under small perturbations, in particular, $M \rightarrow M_{\varepsilon}$. Therefore, we obtain

$M_{\varepsilon}=\left\{(y, I, \gamma, \phi) \mid y=q_{0}=(0,0), I \in\left[I_{1}, I_{2}\right], \gamma \in[0,2 \pi), \phi=\Omega t+\phi_{0}\right\}$.

Considering the following cross-section of the phase space

$$
\Sigma^{\phi_{0}}=\left\{(y, I, \gamma, \phi) \mid \phi=\phi_{0}\right\}
$$

Let the manifold $M_{\varepsilon}^{\phi_{0}}(\phi)$ represent the manifold $M_{\varepsilon}(\phi)$ on the cross-section $\Sigma^{\phi_{0}}$. And restricting the system (8) on the manifold $M_{\varepsilon}^{\phi_{0}}(\phi)$, we have

$$
\begin{gathered}
\dot{I}=-\varepsilon \mu I-2 \varepsilon f I \sin 2 \gamma \cos \phi_{0} \\
\dot{\gamma}=-b--\frac{3}{4} \alpha I-\frac{1}{2} \alpha_{2} y_{1}^{2}-\varepsilon f(\cos 2 \gamma+1) \cos \phi_{0}
\end{gathered}
$$

The $n$-th order energy-difference function for the dissipative case is given as follows:

$$
\begin{aligned}
\Delta^{n} H\left(\gamma, \phi_{0}\right)= & H_{D}\left(h, \gamma+n \Delta \gamma, \phi_{0}\right)-\sum_{j=0}^{N-1} \int_{-\infty}^{\infty} \Omega D_{\phi} H_{D}(h, \gamma+j \Delta \gamma, \phi) d s \\
& -H_{D}\left(h, \gamma, \phi_{0}\right)-\left.\sum_{i=1}^{N} \int_{-\infty}^{\infty}\left\langle D H_{0}(x, I), g(x, I, \gamma, \phi)\right\rangle\right|_{x^{i}(t)} d t
\end{aligned}
$$

Computing (17) leads to the following expression for the dissipative energy difference function

$$
\Delta^{n} H_{D}\left(\gamma, \phi_{0}\right)=f\left[I_{r}(\cos 2(\gamma+n \Delta \gamma)-\cos 2 \gamma) \cos \phi_{0}-\frac{4 n d \eta \Delta \gamma}{3 \alpha_{2}}+\frac{F}{f}\right]
$$

where

$$
\begin{aligned}
F= & \Omega f I_{r} \pi\left[\frac{\Omega(\Delta \gamma)^{3} \sin \phi_{0}}{24 \eta}+\frac{\Delta \gamma \cos \phi_{0}}{\sqrt{\eta}}\right] \csc h\left(\frac{\Omega \pi}{2 \sqrt{\eta}}\right) \cdot \sum_{j=0}^{n-1} \sin 2\left(\gamma_{0}+j \Delta \gamma\right) \\
& -\frac{\Omega^{2}}{2 \eta} f I_{r} \pi(\Delta \gamma)^{2} \sin \phi_{0} \csc h\left(\frac{\Omega \pi}{2 \sqrt{\eta}}\right) \cdot \sum_{j=0}^{n-1} \cos 2\left(\gamma_{0}+j \Delta \gamma\right)
\end{aligned}
$$

Defining a dissipative factor $d=\frac{\mu}{f}$ such that $d$ gives the relative measure of the dissipative effect with respect to the excitation amplitude. Hence the upper bound on the value of the dissipative factor is obtained as follows:

$$
|d|<d_{\max }=\left|\frac{6 \mu \alpha_{2} I_{r} \cos \phi_{0} \sin (n \Delta \gamma)}{3 \alpha_{2} F-4 n \mu \eta \Delta \gamma}\right|
$$


For any s mall dissipative factor $d<1$, we obtain an upper bound on the maximum number of pulses

$$
|n|<n_{\max }=\left|\frac{3 \alpha_{2} I_{r} \cos \phi_{0} \sin (n \Delta \gamma)}{2 d \eta \Delta \gamma}\right|+\left|\frac{3 \alpha_{2} F}{4 \mu \eta \Delta \gamma}\right|
$$

For any $n$ satis fying $n \Delta \gamma \neq 2 l \pi(l=0,1,2, \cdots)$, there are two transverse zeroes of the dissipative energy difference function in the interval $\gamma \in[0, \pi]$, that is,

$$
\begin{gathered}
\gamma_{-, 1}^{n}=\pi-\left[\frac{n \Delta \gamma}{2}+\frac{\alpha_{0}}{2}\right] \bmod \pi \\
\gamma_{-, 2}^{n}=\pi-\left[\frac{\pi}{2}+\frac{n \Delta \gamma}{2}-\frac{\alpha_{0}}{2}\right] \bmod \pi
\end{gathered}
$$

where $\alpha_{0}=\arcsin \left[\frac{F}{2 f I_{r} \cos \phi_{0} \sin (n \Delta \gamma)}-\frac{2 n \eta d \Delta \gamma}{3 \alpha_{2} I_{r} \cos \phi_{0} \sin (n \Delta \gamma)}\right]$.

We now define the set of transveral zeros of $\Delta^{n} H_{D}\left(\gamma, \phi_{0}\right)$ as follows

$$
Z_{-}^{n}=\left\{(h, \gamma) \mid \Delta^{n} H_{D}\left(\gamma, \phi_{0}\right)=0, D_{\gamma} \Delta^{n} H_{D}\left(\gamma, \phi_{0}\right) \neq 0\right\} .
$$

Let us start by defining the energy sequence

$$
h_{0}=H_{D}\left(0, \gamma_{s}\right), h_{n}=\max \left[H_{D}\left(0, \gamma_{-, 1}^{n}\right), H_{D}\left(0, \gamma_{-, 2}^{n}\right)\right]
$$

and the sequence of sets

$$
A_{0}=\phi, A_{n}=\left\{(h, \gamma) \in S_{0} \mid H_{D}\left(h, \gamma, \phi_{0}\right)<h_{n}\right\}, \quad n \geq 1
$$

Next we define the pulse sequence

$$
N_{1}=1, N_{k}=\min \left\{n \in Z \mid n>N_{k-1}, h_{n}>h_{N_{k-1}}\right\}, \quad k \geq 2
$$

Further, we define the layer sequence

$$
L_{N_{k}}=\operatorname{Int}\left(A_{N_{k}} \backslash A_{N_{k-1}}\right)
$$

where $\operatorname{Int}(\cdot)$ refers to the interior of a set. The construction of the layer sequence is shown in Figure 3. And the corresponding inner angular radii of the layers in the layer sequence is defined as

$$
r_{N_{k}}=\min \left(\left|\gamma_{c}-\gamma_{-, 1}^{N_{k}}\right|,\left|\gamma_{c}-\gamma_{-, 2}^{N_{k}}\right|\right)
$$

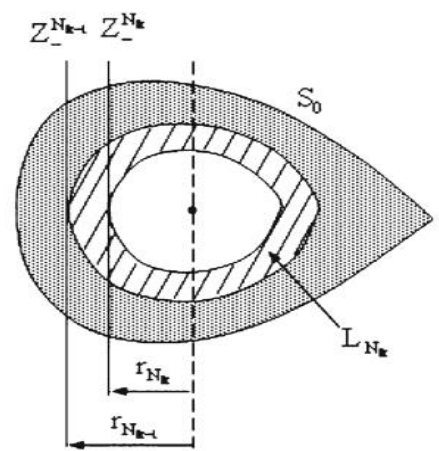

Figure 4 gives the pulse sequence and layer radius sequence as a function of the phase shift in the Hamiltonian case when $N=100$ at the cross-section $\phi=0$. In the left diagram, the horizontal line segments at each level $N$ indicate that an infinity of $N$-pulse orbits exist for all values of the phase shift in the interval below that line. And this diagram shows a fairly stable pulse distribution for lower pulses and increasing sensitivity to small changes in the parameters for higher pulses. In the right diagram, $\Delta \gamma$ can be regarded as an independent bifurcation parameter, and the resulting bifurcation diagram is an infinite binary tree, which can be called the homoclinic tree. The diagram for the layer radii also has a secondary meaning: for fixed $\Delta \gamma,\left\{r_{N_{k}}\right\}$ gives the angular distance of the take-off curves of multi-pulse orbits from the nearest center on the manifold.

Figure 5 gives the pulse sequence and layer radius sequence as a function of the phase shift in the dissipative case when $N=100$ at the cross-section $\phi=0$. The layer radius diagrams now do not refer to layers of periodic orbits, but their secondary meaning remains valid for the full dissipative system: they show the approximate angular distance of the take-off curves of multi-pulse orbits from the nearest sinks on the manifold.
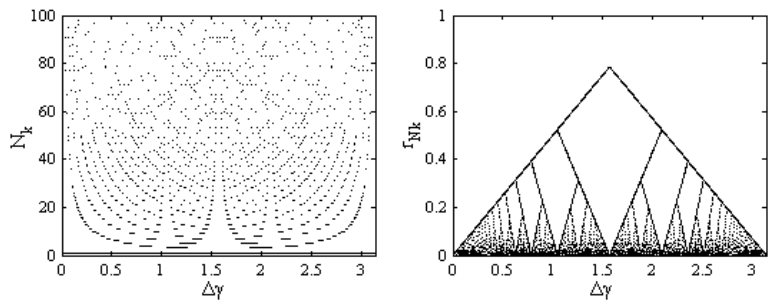

Figure 4 The pulse sequence and the layer sequence as a function of a function of the phase shift in the Hamiltonian case
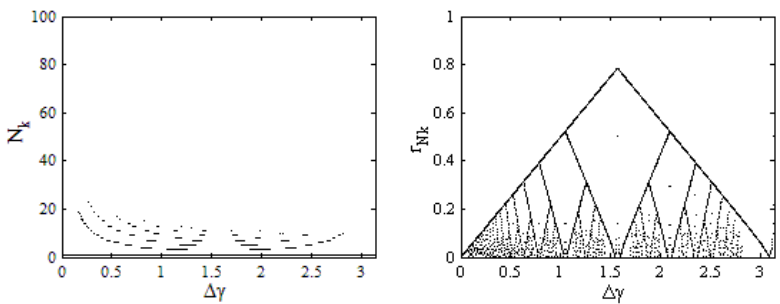

$d=0.001$
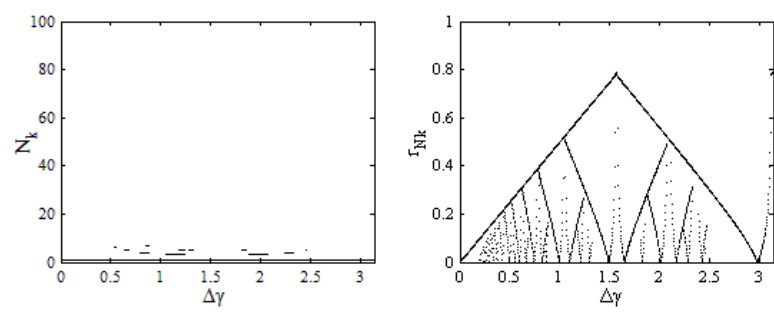

$d=0.01$

Figure 3 The construction of the lay er sequence

However, all these sequence is finite due to (20). For any periodic orbit $\gamma \subset L_{N_{k}}$, the pulse number is $N(\gamma)=N_{k}$. 

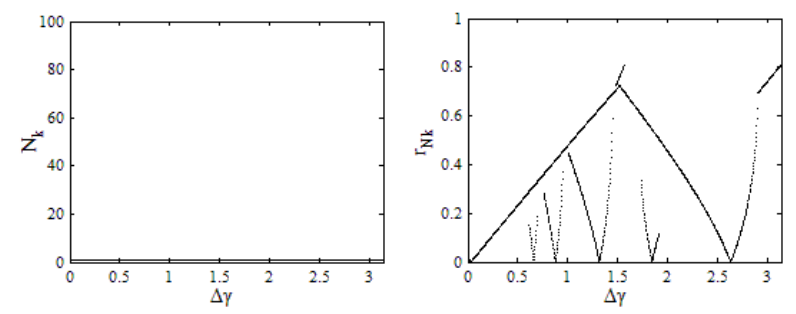

$d=0.1$

Figure 5 The pulse sequence and the lay er sequence as a function of a function of the phase shift in the dissipative case

\section{Conclusion}

In this paper, we have generalized the energy-phase method to deal with the non-autonomous nonlinear system and derive the explicit formulas of the energydifferent function. Applying our method to a two-mode approximation of parametrically excited, simply supported rectangular thin plate, we find the homoclinic tree both in the Hamiltonian case and the dissipative case are shown in Figure 4 and Figure 5. The pulse number diagrams show a variety of ways in which $N$ can change. And the layer radius diagrams gives the layers of periodic orbits, and the angular distance of the take-off curves of multi-pulse orbits from the nearest center on the man ifold in the Hamiltonian case. But the layer radius diagrams in the dissipative case just show the approximate angular distance of the take-off curves of multi-pulse orbits from the nearest sinks on the manifold.

\section{Acknowledgments}

The authors gratefully acknowledge the support of National Natural Science Foundation of China (NNSFC) through grant Nos.11290152 and 11072008, the Funding Project for Academic Human Resources Development in Institutions of Higher Learning under the Jurisdiction of Beijing Municipality (PHRIHLB).

\section{References}

1. G. Haller, S. Wiggins. Phys D, 66(1993).

2. G. Haller. Phys. Lett. A, 200(1995).

3. G. Haller, S. W iggins. Arch. Ration. Mech. and Anal, 130(1995).

4. G. Haller, S. Wiggins. Phys D, 85(1995)

5. G. Haller, S. Wiggins. Phys D, 90(1996).

6. G. Haller. J. S. Phys, 86(1997).

7. G. Haller. Commun. Math. Phys, 193(1998).

8. G. Haller. Commun. Appl. Math, LII(1999).

9. G. Haller, G. Menon. Phys. Lett. A, 263(1993).

10. G. Haller. Chaos near resonance. SpringerVerlag(1999).

11. W. Zhang. J. Sound Vib, 239(2001). 